\title{
TURISMO ACCESIBLE COMO EJEMPLO DE RESPONSABILI- DAD SOCIAL EN LAS EMPRESAS Y DESTINOS TURÍSTICOS. EL CASO DE LOUSÃ (PORTUGAL)
}

\author{
Elisabeth Kastenholz
}

Universidade de Aveiro

María de las Mercedes Galán-Ladero²

Universidad de Extremadura

\begin{abstract}
Resumen: La responsabilidad social, entendida como una nueva cultura empresarial basada en la gestión ética, puede servir como cauce para mejorar la competitividad y la reputación de las empresas turísticas, respondiendo así a las crecientes demandas de la sociedad en general y de los consumidores en particular. En este contexto surge el concepto de "turismo sostenible", que trata de ofrecer una experiencia digna y enriquecedora al turista, al mismo tiempo que trata de responder a la creciente preocupación por los impactos negativos del turismo en las áreas de destino. El "turismo accesible" se encuadra en este marco, una modalidad de oferta turística integrada que permite a todos disfrutar de experiencias turísticas "sin barreras". Esta modalidad de turismo no sólo permite a las empresas una especialización ( $y$, por tanto, una mejor satisfacción del mercado de personas con discapacidad), sino también les permite mostrar una imagen más humana, conseguir una reputación de empresa turística socialmente responsable, lo cual puede constituir una importante ventaja competitiva. En este artículo nos centramos en el caso de Lousã, un municipio portugués que ha apostado por el turismo accesible como estrategia de cualificación y diferenciación del destino, como estrategia de desarrollo y posicionamiento en un entorno crecientemente competitivo. Esta apuesta ha dinamizado numerosas iniciativas y nuevos caminos de colaboración entre diversos agentes (públicos y privados), ofreciendo oportunidades muy interesantes en un contexto de desarrollo de los territorios que busca no excluir a nadie.
\end{abstract}

Palabras clave: Responsabilidad Social, Turismo Sostenible, Turismo Accesible, marketing de destinos turísticos, Lousã.

Resumo: $O$ turismo accesible como exemplo de responsabilidade social nas empresas e destinos turísticos. O caso de Lousã (Portugal)

1 Departamento de Economia, Gestão e Engenharia Industrial (DEGEI) - Universidade de Aveiro. Campus Universitário de Santiago, 3810-193-Aveiro (Portugal). E-mail: elisabethk@.ua.pt

2 Departamento de Dirección de Empresas y Sociología - Universidad de Extremadura. Facultad de CC. Económicas y Empresariales, Avda. Elvas s/n, 06071-Badajoz (España).E-mail: mgalan@unex.es 
A responsabilidade social, entendida como unha nova cultura empresarial baseada na xestión ética, pode servir como canle para mellorar a competitividade e a reputación das empresas turísticas, en resposta ás crecentes demandas da sociedade en xeral e dos consumidores en particular. Neste contexto xorde o concepto de turismo sustentable, que trata de ofrecerlles unha experiencia digna e enriquecedora aos turistas e, asemade, responder á crecente preocupación polos impactos negativos do turismo nas áreas de destino.

O turismo accesible encádrase neste marco como unha modalidade de oferta turística integrada que lles permite a todas as persoas gozar de experiencias turísticas «sen barreiras». Esta modalidade de turismo non só fai posible a especialización das empresas (e, por tanto, que satisfagan mellor o mercado de persoas con minusvalías), mais tamén estas mostraren unha imaxe máis humana e conseguiren unha reputación de empresa turística socialmente responsable, o cal pode constituír unha importante vantaxe competitiva.

Neste artigo centrámonos no caso de Lousã, un municipio portugués que apostou polo turismo accesible como estratexia de cualificación e diferenciación do destino, como estratexia de desenvolvemento e posicionamento nun ámbito crecentemente competitivo. Esta aposta dinamizou numerosas iniciativas e novos camiños de colaboración entre diversos axentes públicos e privados, as cales lle permiten ofrecer oportunidades moi interesantes nun contexto de desenvolvemento dos territorios que busca non excluír a ninguén.

Palabras clave: responsabilidade social, turismo sustentable, turismo accesible, mercadotecnia de destinos turísticos, Lousã.

\begin{abstract}
Accessible tourism as an example of social responsibility in tourism companies and destinations. The case of Lousã (Portugal).

Social responsibility, understood as a new business culture based on ethical management, may serve as a route for improving competitiveness and the reputation of tourism companies, thus responding to the growing demands of society in general and of consumers in particular. The concept of "sustainable tourism" arises within this context. It aims to offer the tourist a worthy and enriching experience, and at the same time to respond to the growing concern about the negative impact of tourism on the destination areas. "Accessible tourism" fits within this framework, a type of integrated tourism which allows everybody to enjoy tourism experiences "without barriers".This type of tourism not only allows companies a specialisation (and, therefore, better satisfaction of the disabled person market), but it also allows them to show a more humane image and to achieve a reputation as a tourism company which is socially responsible, which may constitute a significant competitive advantage. This article focuses on the case of Lousã, a Portuguese town committed to accessible tourism as a strategy for qualifying and differentiating the destination, as a strategy for developing and positioning in an increasingly competitive environment. This commitment has revitalised numerous initiatives and new types of collaboration between different agents (public and private), offering very interesting opportunities in the context of developing territories which aim not to exclude anybody.

Key words: Social Responsibility, sustainable tourism, accessible tourism, marketing of tourist destinations, Lousã.
\end{abstract}

\title{
1.- INTRODUCCIÓN.
}

El entorno, cada vez más dinámico y globalizado, provoca en las organizaciones una presión importante de sus competidores, incrementa la dificultad para la diferenciación de los productos (aumentando su complejidad y la exigencia de una mayor calidad), establece una tendencia de precios a la baja (estrechando los márgenes), aparece una saturación de los medios de comunicación (disminuyendo la eficacia publicitaria) y ocasiona un incremento en los costes promocionales. 
Por otra parte, el perfil del nuevo consumidor es el de una persona más formada, informada, sofisticada, exigente y escéptica, menos fiel a las marcas y con una estructura de valores distinta (Galán et al, 2006), más sensibilizado con el entorno, lo que le lleva a incorporar consideraciones sociales, medioambientales o éticas en los procesos de selección de productos y marcas (fenómeno conocido como "consumo responsable").

La expansión del consumo responsable, en sus distintas modalidades, es un factor que presiona a las empresas para que adopten prácticas de responsabilidad social corporativa (Nieto y Fernández, 2004). Así, existe una tendencia creciente a que la sociedad en general, y los consumidores en particular, exijan a la empresa conductas cada vez más responsables y transparentes (Ruano y Rojas, 2006).

Por otro lado, el éxito de las empresas está de alguna manera relacionado con el éxito de las comunidades en las que se desenvuelven (Ruano y Rojas, 2006): influyen en las comunidades en las que se desarrollan tanto interna (empleados) como externamente (consumidores y entorno). Esto es verdaderamente relevante en el caso de las empresas turísticas, cuyo éxito depende directamente del atractivo global de los destinos turísticos en los que se localizan, funcionando como un sistema (Leiper, 1979; Gunn, 1994) activado por los turistas (Gnoth, 2003), donde la población local y el entorno (tanto físico-natural como socio-cultural) desempeñan un papel fundamental.

En este contexto, las empresas se ven obligadas a buscar nuevas estrategias para posicionar sus productos. La innovación y la ventaja competitiva pueden resultar de incluir consideraciones sociales y medioambientales en la estrategia empresarial. Por todo ello, la tendencia actual, a nivel mundial, se dirige hacia un mayor énfasis de la responsabilidad social corporativa (RSC), considerándola desde un punto de vista estratégico.

Esta nueva perspectiva de gestión estratégica se aplica igualmente a las empresas del sector turístico, donde el desarrollo sostenible asume una posición paradigmática de reconocimiento creciente (Bramwell y Lane, 1993). También el fenómeno del turismo accesible ilustra esa nueva visión de responsabilidad social, valorada por el mercado e inductor de una nueva imagen, identidad y dinámica a nivel de destino, como se muestra en el caso de Lousã, un destino turístico de pequeñas dimensiones que apuesta por el turismo accesible como estrategia de desarrollo y posicionamiento en un entorno crecientemente competitivo.

\section{2.- LA RESPONSABILIDAD SOCIAL COMO NUEVO PARADIGMA DEL MUNDO EMPRESARIAL. ESPECIAL RELEVANCIA PARA LOS SERVICIOS.}

La RSC es una nueva forma de pensar, una nueva cultura empresarial basada en la gestión ética y en la responsabilidad de la empresa ante la sociedad como cauce para mejorar la competitividad y la reputación de las empresas al tiempo que cubren las demandas sociales en estos aspectos (Forética, 2002).

La responsabilidad social de las empresas se define como "la integración voluntaria, por parte de las empresas, de las preocupaciones sociales y medioambientales en sus operaciones comerciales y sus relaciones con sus interlocutores" (Comisión de las 
Comunidades Europeas, 2001: 7). Kotler y Lee (2005: 3) también destacan el carácter voluntario del compromiso de mejorar el bienestar de la comunidad: "la responsabilidad social corporativa es un compromiso para mejorar el bienestar de la comunidad (incluye las condiciones humanas así como los asuntos medioambientales) a través de prácticas empresariales discrecionales (compromiso voluntario) y contribuciones de los recursos corporativos".

El concepto de RSC descansa en dos premisas fundamentales (Wartick y Cohnran, 1985): la empresa existe para "deleitar" a la sociedad, y la empresa actúa como un agente moral con la sociedad (las compañías reflejan y refuerzan valores). Además, hay que destacar que la RSC tiene un carácter multidimensional.

El razonamiento sistemático sobre el marco conceptual de la RSC comenzó en Estados Unidos a mediados del siglo XX (Carroll, 1999). En 1953, Bowen abrió el debate "moderno" sobre RSC (Wartick y Cohnran, 1985), pero no fue hasta la década de los setenta cuando adquirió una mayor relevancia, al surgir opiniones divergentes sobre si una corporación puede (o no) tener una responsabilidad moral, si puede tener responsabilidades sociales (Crane y Matten, 2007), lo que ha llevado a plantear enfoques distintos (Bueno, 2001; Suter, 1995):

- Enfoque negativo (o crítico): muestra una oposición a incrementar la responsabilidad social de la empresa más allá de lo que representa cumplir con su objetivo primordial que es obtener el máximo beneficio ("máximo retorno para los accionistas") y demostrar que es competitiva. En esta postura destacó Milton Friedman (1970), que basaba su argumentación en tres premisas principales (Crane y Matten, 2007): 1) sólo las personas pueden tener responsabilidades, 2) es responsabilidad de los directivos actuar únicamente según los intereses de los accionistas (conseguir beneficios) y 3) los problemas y asuntos sociales son competencia propia del Estado (y no de los directivos de la empresa).

- Enfoque positivo (o favorable): acepta un mayor compromiso social como forma de incrementar los beneficios a largo plazo y facilitar el crecimiento de la empresa. Los valores y creencias de la cultura organizacional tienen una fuerte influencia en el comportamiento y en la toma de decisiones éticas del individuo (en Crane y Matten, 2007). En este enfoque destacó Christopher Stone, que refutó los argumentos de Friedman (Suter, 1995). Esta postura desarrolla determinadas políticas de mecenazgo o de obra social y cultural.

- Enfoque intermedio: acepta la responsabilidad social como estrategia de promoción de la imagen corporativa de la empresa, por lo que es importante una adecuada negociación con los grupos de interés (stakeholders), especialmente públicos y sociales. De esta forma, se pueden asegurar tanto los resultados a corto como a largo plazo. Sin cuestionar la primacía de los intereses de los propietarios, tiene en cuenta a todos los partícipes o interesados en la compañía (stakeholders), tratando de equilibrar los intereses de todos los grupos. De esta forma, la RSC se presenta como un modelo emergente y alternativo de gestión que reco- 
noce a la empresa como un entramado de relaciones, no sólo de propietarios (accionistas) sino de todas aquellas partes o grupos interesados en la existencia y marcha de la empresa: empleados, clientes, proveedores, competidores, comunidad local, administración pública, medio ambiente y sociedad en general (Lizcano, 2006).

En sus inicios, la responsabilidad social fue fomentada, fundamentalmente, por las grandes empresas o sociedades multinacionales. Sin embargo, la responsabilidad social es aplicable a cualquier tipo de empresa y en todos los sectores de actividad ${ }^{3}$. De esta forma, aunque las empresas tienen por objeto generar beneficios económicos, pueden también contribuir al mismo tiempo al logro de objetivos sociales y medioambientales, integrando la responsabilidad social como inversión estratégica en el núcleo de su estrategia empresarial, sus instrumentos de gestión y sus actividades (Comisión de las Comunidades Europeas, 2001). Por tanto, es importante la extensión de las prácticas de RSC a todo el tejido empresarial (Puerta, 2006).

Las prácticas responsables tendrán éxito y se afianzarán cuando los valores aplicados por las empresas estén alienados con los valores de los consumidores y éstos realmente así lo reconozcan ejercitando sus opciones de elección a través de instrumentos como la inversión socialmente responsable o las propias pautas de consumo (Puerta, 2006).

La justificación de la aplicación de la RSC se basa en las ventajas que puede ocasionar. Las empresas consideran que ser socialmente responsables podría ser recompensado con (Crane y Matten, 2007; Arenas, 2006; Córdoba, 2006; Viñets, 2006): más clientes (y más satisfechos, leales e identificados con la empresa, con una elección de consumo basada en criterios de mayor implicación emocional); empleados más motivados y comprometidos (menos absentismo y rotación, mayor productividad y lealtad), orgullosos de los valores de RSC que existen en la cultura empresarial; mayor facilidad de acceso a la financiación (aumenta su credibilidad, lo que atrae a nuevos inversores ${ }^{4}$ ); una mayor independencia corporativa del gobierno y mejores relaciones con la Administración (también puede suponer beneficios fiscales, disminución del riesgo de sanciones y sinergias en áreas diversas, como empleo o seguridad e higiene en el trabajo); una comunidad más segura, mejor educada y más equitativa, lo cual, consecuentemente, beneficia a la corporación creando un contexto estable y mejorado en el cual hacer negocio. Hacer una contribución positiva a la sociedad podría ser considerada como una inversión a largo plazo.

En definitiva, las prácticas de RSC mejoran las condiciones del entorno en el que operan las empresas, reducen riesgos y aumentan su reputación (Nieto y Fernández,

\footnotetext{
3 En Europa, esto es especialmente relevante si se quiere alcanzar la estrategia de desarrollo sostenible, porque la RSC es una demanda social y del propio mercado (Escudero, 2006).

4 Los grandes proveedores de índices bursátiles, como FTSE o Dow Jones, han comenzado a ofrecer nuevas series de índices, como FTSE 4 Good o Dow Jones Sustainability Index, que incluyen sólo a aquellas empresas que cumplen unos estrictos criterios de RSC. Las compañías que consiguen superar los filtros establecidos ven reforzada su reputación y, en muchas ocasiones, consiguen un acceso más favorable a las fuentes de financiación (Nieto y Fernández, 2004).
} 
2004). La RSC puede ser un instrumento estratégico para lograr ventajas competitivas, especialmente en sectores de alto crecimiento (McWilliams y Siegel, 2001). Puede aumentar la inversión en $\mathrm{I}+\mathrm{D}$ (en los procesos, al llevarlos a cabo de una manera socialmente responsable; o en la innovación de productos, incorporando atributos socialmente responsables), facilitar la entrada en otros mercados, conseguir una diferenciación comercial y una vinculación emocional con los diversos stakeholders (clientes, principalmente).

En 1979, Carroll estableció un modelo ${ }^{5}$ con las diferentes dimensiones de la RSC (Carroll, 1999):

- Responsabilidades económicas (requeridas por la sociedad). La primera responsabilidad de una empresa es ser propiamente una unidad económica (funcionar y estar en el negocio). Es la base de todas las demás responsabilidades.

- Responsabilidades legales (requeridas por la sociedad). Las empresas deben respetar la ley y seguir las reglas del juego.

- Responsabilidades éticas (esperadas por la sociedad). Obligan a que las empresas hagan lo que es correcto y justo, incluso cuando no están obligadas a hacerlo así por el marco legal.

- Responsabilidades discrecionales o filantrópicas (deseadas por la sociedad). Actividades que son a discreción de la empresa, para mejorar la calidad de vida de los empleados, de las comunidades locales y de la sociedad en general.

Además, las estrategias de RSC pueden ser, según la sensibilidad de la empresa a los asuntos sociales (Carroll, 1999; en Crane y Matten, 2007), de:

- Reacción: la empresa niega cualquier responsabilidad en temas sociales.

- Defensa: la empresa admite cierta responsabilidad, pero hace lo mínimo requerido.

- Acomodación: la corporación acepta la responsabilidad y hace lo que demandan sus stakeholders (grupos de interés).

- Proacción: la empresa busca ir más allá de las normas del sector y se anticipa a las expectativas futuras, haciendo más de lo esperado.

Por otra parte, la RSC también debe ser considerada desde dos niveles (en Escudero, 2006):

- Un nivel interno: mejoras que se pueden hacer relacionadas con la organización interna de la empresa (por ejemplo, conciliación laboral y familiar, con mejora de las condiciones de trabajo). Se debería empezar por este nivel.

5 Este modelo ha sido ampliamente aceptado en el ámbito académico. No obstante, presentaba algunas limitaciones (Crane y Matten, 2007): no decía nada sobre lo que podría ocurrir si dos o más responsabilidades entraban en conflicto (por ejemplo, responsabilidades éticas y económicas) y, además, estaba sesgado (dirigido al contexto de Estados Unidos). 
- Un nivel externo: con dos subniveles. Uno, inmediato o cercano (mejoras dirigidas a proveedores, clientes, competencia, mercado y administración pública); otro, más amplio (mejoras dirigidas al entorno social y medioambiental).

Un aspecto adicional a destacar es que la RSC requiere la implicación de toda la empresa. Es necesario el compromiso de todas las personas de la empresa, en todos los niveles de la organización, y su integración en la estrategia y la misión corporativa (Arenas, 2006).

Los primeros que han aplicado RSC han logrado una ventaja competitiva importante (al principio, la RSC se consideraba una innovación, lo que suponía una ventaja competitiva para las compañías "emprendedoras”). Sin embargo, al generalizarse, sólo sirve para descalificar a las que no la practiquen (Córdoba, 2006).

Al mismo tiempo, han surgido numerosas iniciativas dirigidas a establecer una serie de principios y códigos de conducta con el fin de estimular y orientar a las empresas en la adopción de criterios de RSC (Nieto y Fernández, 2004; Forética, 2002). También han aparecido índices y clasificaciones que juzgan el comportamiento social de las empresas.

Existe un gran número de estándares y una gran heterogeneidad de intereses de las instituciones que los han propuesto, pero todos ellos se han concebido con el propósito de ayudar a las empresas a gestionar sus acciones en materia de RSC y a facilitar la medición de sus resultados (Nieto y Fernández, 2004). Los estándares de RSC más comunes son (Arenas, 2006): el Global Reporting Initiative (GRI), con directrices para la elaboración de memorias de sostenibilidad; el $S A$ 8000, una certificación auditable basada en las normas internacionales del trabajo; la AA 1000 Assurance Standard, una guía para involucrar a grupos de interés en los indicadores y la comunicación de la RSC; la ISO 26000, una guía no certificable para la aplicación voluntaria de la RSC (en elaboración); el UN Global Compact, con principios generales en los que las empresas se comprometen a mostrar progreso; el Ethical Trading Initiative Base Code, un código sobre normas internacionales de trabajo; o la $S G E 21$, una norma de empresa que enfoca por áreas de gestión los planteamientos éticos en las organizaciones.

Sin embargo, actualmente no existe consenso sobre cuáles son los principios y las normas que debe adoptar una empresa para que pueda ser considerada socialmente responsable.

En cuanto a las principales iniciativas dentro de la RSC, Kotler y Lee (2005) señalan 6:

- Promociones de causas: cuando una corporación proporciona fondos, contribuciones en especie u otros recursos corporativos para incrementar la conciencia y la preocupación sobre una causa social o para apoyar el fundraising, la participación o la captación de voluntarios para una causa. La empresa puede iniciar y dirigir la promoción por sí misma, puede tener un socio principal (una organización no gubernamental - ONG, por ejemplo), o puede ser uno de los diferentes patrocinadores del evento. 
- Marketing con causa: una empresa se compromete a hacer una contribución o a donar un porcentaje de los ingresos a una causa específica, basada en las ventas (o uso) de un producto. Generalmente, esta oferta es por un determinado periodo de tiempo, para un producto específico y para una ONG específica. En este escenario, la compañía está frecuentemente asociada a una organización no lucrativa (ONL), creando una relación mutuamente beneficiosa, diseñada para incrementar las ventas de un producto particular y para generar apoyo financiero para la ONG. Proporciona también a los consumidores una oportunidad para contribuir a sus ONG favoritas.

- Marketing Social Corporativo: una empresa apoya el desarrollo y/o implementación de una campaña de cambio de comportamiento que intenta mejorar el medio ambiente o el bienestar de la comunidad. El hecho distintivo es el enfoque en el cambio de comportamiento. Una organización puede desarrollar e implementar una campaña de cambio de comportamiento por sí misma, aunque frecuentemente lo hace asociándose con agencias del sector público y/o ONL.

- Filantropía corporativa: una empresa hace una contribución directa a una ONG o causa, frecuentemente bajo la forma de cantidades (subvenciones) en efectivo, donaciones, y/o servicios en especie. Esta iniciativa es, posiblemente, la más tradicional de todas las iniciativas sociales.

- Voluntariado de la comunidad: una empresa proporciona servicios de voluntariado a la comunidad - apoya y anima a que los empleados, distribuidores y/o miembros franquiciados dediquen voluntariamente su tiempo a apoyar causas y organizaciones de la comunidad local. Esta actividad puede ser un esfuerzo autónomo o puede ser hecho en asociación con una ONG. Las actividades de voluntariado pueden ser organizadas por la empresa, o los empleados pueden elegir sus propias actividades y recibir apoyo de la compañía (por ejemplo, a través de tiempo pagado sin trabajar en la empresa pero empleado en la actividad seleccionada).

- Prácticas empresariales socialmente responsables: una empresa adopta y conduce prácticas empresariales discrecionales e inversiones que apoyan causas sociales para mejorar el bienestar de la comunidad y proteger el entorno. Las iniciativas pueden ser concebidas e implementadas por la organización o puede ser en alianza con otras.

En definitiva, la RSC permite conseguir el denominado "círculo virtuoso" (figura 1): las acciones de responsabilidad social mejoran el clima laboral y los empleados atienden mejor a los clientes. Estos últimos, se sienten más satisfechos y vinculados emocionalmente a la empresa, incrementando sus compras, lo que repercute en unos mayores beneficios para la compañía, que atraen el interés de los inversores, proveedores, distribuidores, etc. Y si la empresa va bien, se asegura la estabilidad en el empleo, lo que permite cerrar el círculo. 
Figura 1: Círculo virtuoso

\section{Cliente}

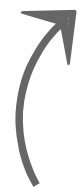

Empleado

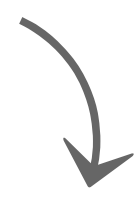

Inversor

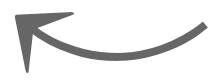

Fuente: Elaboración propia.

\section{3.- LA RESPONSABILIDAD SOCIAL EN LAS EMPRESAS DEL SECTOR TURÍSTICO.}

La RSC y el consumo responsable señalados anteriormente son especialmente relevantes en el sector turístico (Henderson, 2007; Nicolau, 2008). Desde hace algunos años, los turistas se interesan cada vez más por la situación de las zonas que visitan y las consecuencias que ese turismo puede tener para el medio ambiente y para la población local (por ejemplo, explotación del entorno natural, cambio de costumbres locales, etc.). Así, han surgido diversas modalidades, a veces poco diferenciadas, como el denominado "turismo sostenible", el "ecoturismo", el "turismo soft" o el "turismo responsable" (Bramwell y Lane, 1993; Middleton y Hawkins, 1998). Actualmente, uno de los ejemplos más emblemáticos de la nueva actitud de los turistas es el llamado "turismo solidario", mediante el cual los turistas tratan de descubrir la situación real de los países que visitan, alejándose de los típicos circuitos tradicionales y conociendo los distintos proyectos de desarrollo llevados a cabo en la zona por las ONG u organismos internacionales (McGehee y Santos, 2005).

El turismo sostenible permite añadir nuevos valores y atributos emocionales, mejorando la satisfacción de los visitantes y contribuyendo al desarrollo local de una manera positiva. De acuerdo con la Organización Mundial del Turismo (OMT, 2004), "el turismo sostenible atiende a las necesidades de los turistas actuales y de las regiones receptoras y, al mismo tiempo, protege y fomenta las oportunidades para el futuro. Se concibe como una vía hacia la gestión de todos los recursos, de forma que puedan satisfacerse las necesidades económicas, sociales y estéticas, respetando al mismo tiempo la integridad cultural, los procesos ecológicos esenciales, la diversidad biológica y los sistemas que sostienen la vida". Estos principios son "aplicables a todas las formas de turismo, en todos los tipos de destinos (incluido el turismo de masas)..." y se refieren "a los 
aspectos ambientales, económicos y socioculturales del desarrollo turístico, habiéndose de establecer un equilibrio adecuado entre esas tres dimensiones para garantizar su sostenibilidad a largo plazo" (OMT, 2004).

En ese contexto, el turismo sostenible debe, de acuerdo con la misma fuente (OMT, 2004):

1) Dar un uso óptimo a los recursos ambientales, que son un elemento fundamental del desarrollo turístico, manteniendo los procesos ecológicos esenciales y ayudando a conservar los recursos naturales y la diversidad biológica;

2) Respetar la autenticidad sociocultural de las comunidades anfitrionas, conservar sus activos culturales, arquitectónicos y monumentales, así como sus valores tradicionales, y contribuir al entendimiento y tolerancia interculturales;

3) Asegurar unas actividades económicas viables a largo plazo, que reporten a todos los agentes unos beneficios socioeconómicos bien distribuidos, entre los que se cuenten oportunidades de empleo estable y de obtención de ingresos y servicios sociales para las comunidades anfitrionas, y contribuyendo a la reducción de la pobreza;

4) Proporcionar también un alto grado de satisfacción a los turistas y representar para ellos una experiencia significativa, que los haga más conscientes de los problemas de la sostenibilidad y fomente en ellos unas prácticas turísticas sostenibles;

Y todo ello a través de un proceso que asegure la participación informada de todos los agentes relevantes, así como un liderazgo político firme para lograr una colaboración amplia y establecer un consenso. Ese proceso continuo requiere un seguimiento constante de los impactos, para introducir las medidas preventivas o correctivas que resulten necesarias.

Efectivamente, hay un número creciente de empresas en el sector turístico que adoptan una estrategia y una visión de responsabilidad social, abarcando los aspectos económicos, sociales, culturales y ambientales, fomentando un desarrollo sostenible de su negocio y del destino del que forman parte $^{6}$. Y no siendo, por tanto, solamente el ánimo de lucro lo que motiva, en primer lugar, sus acciones, su planificación, su gestión y/o su marketing.

En consecuencia, la evolución más reciente del denominado societal marketing y del marketing no lucrativo hacia una mayor responsabilidad social corporativa, es particularmente adecuada en el campo del turismo (Kastenholz y Paul, 2004; Kastenholz, 2005; Middleton y Hawkins, 1998; Krippendorf, 1989).

En concreto, el dominio de la planificación, gestión y marketing de destinos turísticos, Ashworth y Voogd (1994) sugieren abordarlo como un "proceso por el cual las actividades locales están relacionadas, en la medida de lo posible, con los deseos de los consumidores objetivos. La intención es maximizar el funcionamiento eficiente a nivel

6 Ver ejemplos de Sustainable Travel International: http://www.sustainabletravelinternational.org/ 
social y económico del área en cuestión, de acuerdo con los objetivos más ampliamente definidos". Efectivamente, la integración del turismo en la base económica y sociocultural del destino es una condición importante para permitir una estrategia de turismo sostenible, un turismo con futuro, basado en un desarrollo del sector turístico conciliado con los variados intereses de la comunidad receptora (De Kadt, 1979).

La particularidad del "producto turístico" y las implicaciones de su venta o consumo en los destinos sugieren procedimientos específicos así como una mayor preocupación por los recursos atractivos fundamentales del "producto - destino", al mismo tiempo que se busca la satisfacción del turista y de los objetivos de la población residente. Al comprender los diversos segmentos del mercado, sus perfiles, motivaciones y comportamientos, los agentes turísticos responsables del desarrollo del destino pueden optar por atraer y satisfacer solamente a aquéllos que más les interesan, buscando el referido desarrollo sostenible (Kastenholz, 2004). De este modo, el destino podrá intentar no sólo organizar la oferta sino también "dirigir la demanda", controlando los flujos turísticos para optimizar los resultados de la actividad turística a nivel del destino.

Éste ha sido el caso de un grupo poco atendido en el pasado por la industria del turismo: los turistas con dificultades de movilidad (Daruwalla y Darcy, 2005), un segmento de creciente importancia y con los mismos derechos para ir y disfrutar de vacaciones, pero caracterizado por condicionantes especiales que hacen sus viajes más difíciles. Atender esos condicionantes y esas necesidades especiales puede ser una forma de diferenciación que no atrae solamente a ese grupo de turistas sino que permite igualmente crear una imagen de responsabilidad social que atrae a otros grupos y que, además, tiene otras implicaciones a nivel de la propia comunidad receptora, como se explicará más adelante.

\section{4.- CONCEPTO DE TURISMO ACCESIBLE.}

De acuerdo con el English Tourism Council (2000) "el turismo accesible se refiere a un conjunto de servicios e infraestructuras capaces de garantizar a las personas con necesidades especiales disfrutar sus vacaciones y tiempos de placer sin barreras ni problemas específicos". Se trata, por tanto, de ofrecer servicios o, más concretamente, oportunidades para disfrutar experiencias turísticas, atendiendo a las necesidades específicas de una persona que “...sufre algún tipo de limitación en su capacidad relacional y presenta necesidades especiales durante el viaje, en el alojamiento o en otros servicios turísticos. Incluye esencialmente a personas con discapacidades físicas, sensoriales o intelectuales, así como otras que se encuentren en condiciones de salud y de edad que requieren cuidados especiales, temporal o permanentemente” (OMT, 2005).

Según esta misma fuente, el grupo de personas con algún tipo de discapacidad abarca ya aproximadamente a 50 millones de personas en Europa (es decir, un 10\% de la población), estimándose que en uno de cada cuatro hogares europeos hay una persona con alguna discapacidad. A nivel mundial, el número de personas con deficiencias se estima en 600 millones (Fujiura y Rutkowskikmitta, 2001). Si consideramos además las 
ya ampliamente conocidas tendencias demográficas de envejecimiento de las sociedades occidentales (constituyendo, por cierto, los principales mercados emisores en turismo), y si contabilizamos también a las personas que acompañan los individuos con alguna discapacidad (Buhalis et al, 2005), no quedan dudas sobre la relevancia actual y futura de este grupo de personas como segmento de mercado turístico ${ }^{7}$.

Devile (2007) sugiere que este grupo, en el fondo, incluye a personas que, por motivos diversos, presentan dificultades de movimiento, como padres con cochecitos de bebés y hasta personas con equipaje pesado. Esta definición más amplia de la persona con necesidades especiales nos lleva a concluir que cualquier persona puede estar, en algún momento de su vida, transitoria o más permanentemente, incluida en este grupo. Además de eso, y de acuerdo con Buhalis et al (2005), el concepto de turismo accesible se extiende además a todas aquellas personas que están, directa o indirectamente, afectadas por un familiar o amigo en estas condiciones, ya que sus vacaciones quedan naturalmente condicionadas por esta persona cercana cuando pretenden disfrutar de su compañía.

El turismo accesible, también denominado "Turismo para Todos", puede ser considerado una forma de turismo social, pues procura vencer obstáculos que impiden a un individuo ejercer su derecho de hacer turismo, de viajar, de conocer otras regiones y países, siendo éste un derecho considerado universal según el parecer del Comité Económico y Social Europeo sobre el "Turismo Social en Europa" (2006/C 318/12). Además, puede ser entendido como una fuerza social promotora de inserción, tolerancia y paz (D’Amore, 1988; Higgins-Desbiolles, 2006).

Por otra parte, y de acuerdo con la visión de la Cooperativa Nacional ${ }^{8}$ de Apoyo a Deficientes (CNAD, 2003), el "Turismo Accesible" se encuadra claramente en una perspectiva de "Turismo de Calidad", al exigir que la oferta turística tenga en consideración no sólo la simple propuesta de las condiciones naturales para uso y disfrute del visitante, sino que procure efectivamente la satisfacción de las necesidades de los potenciales clientes, considerando sus respectivas exigencias, condicionantes de viaje y deseos de los mismos. Este requerimiento corresponde, en el fondo, a la perspectiva de marketing que se centra en alcanzar los objetivos de una organización (el lucro y/u otros objetivos, dependiendo de la organización) a través de la satisfacción de las necesidades de su público (Lambin, 2000). Para el público (o segmento de mercado) en cuestión, la exigencia de calidad presupone accesibilidad total, no sólo a nivel físico sino también cognitivo y sensorial, permitiendo una experiencia turística global de calidad.

De este modo, la aplicación de los requisitos de turismo accesible (por ejemplo, a través del "Design for All" en la concepción de los productos y servicios turísticos para lograr la accesibilidad universal) puede venir a representar una importante ventaja competitiva para todos los agentes turísticos que apuesten por esta estrategia.

7 Según la World Health Organization - WHO (2007), en 2020 habrá un grupo de 1.200 millones de personas con más de sesenta años de edad, siendo este fenómeno más acentuado en los principales mercados emisores. 8 Portuguesa. 
Teóricamente, y según la definición de las estrategias de base sugeridas por Michael Porter (1985), la estrategia aquí señalada puede ser considerada como de concentración o enfoque, centrada en un solo mercado objetivo (Lambin, 2000), a través de una especialización de la oferta y de las acciones de marketing perfectamente adaptadas al grupo en cuestión. En este ámbito, merece destacarse que este grupo de turistas revela un alto nivel de fidelización (Burnett y Baker, 2001), ya que, satisfechos, tienden a regresar a aquellos destinos que les permiten disfrutar de experiencias turísticas sin barreras.

Por otro lado, y según la misma clasificación de opciones estratégicas, podrá igualmente constituirse como estrategia de diferenciación, en la medida en que permite a los agentes de la oferta distinguirse de la concurrencia a través de una oferta más cualificada que admite tanto mantener a los otros segmentos de mercado como integrar a un grupo más con necesidades especiales.

Finalmente, consideramos que estas ofertas diferenciadas no deben serlo, esencialmente, por su contenido ni necesariamente por una diferenciación en el espacio y/o en el tiempo, porque este procedimiento podría inducir a una segregación que se opone claramente a los objetivos del "turismo accesible" o "turismo para todos", que busca precisamente la integración de este grupo y no su exclusión y/o su satisfacción aparte.

Si esta estrategia fuera adoptada a nivel de destino, podría permitir la creación de una imagen de marca distintiva, ya que la mayor accesibilidad del destino potenciaría prácticas turísticas más diversificadas y satisfactorias para todos. Podría llevar, por tanto, a un posicionamiento diferenciado en los mercados turísticos en los que el destino tradicionalmente actúa. A través de esta actuación, podría así también no solamente ganar una ventaja competitiva en el segmento de mercado de personas con deficiencias motoras y sus acompañantes de viaje, sino igualmente en los de los diversos públicos y stakeholders del destino turístico en cuestión (residentes, inversores, agentes públicos, visitantes actuales, etc.). De esta forma, el destino mostraría una cara más "humana", una responsabilidad social que merecería ser destacada y reconocida, y que podría llevar a una mayor identificación con el mismo, a una afectividad y lealtad creciente, tal y como acontece en el caso de empresas que proyectan una imagen de responsabilidad social.

\section{5.- EL CASO LOUSÃ.}

Un ejemplo de las preocupaciones a nivel de accesibilidad en el turismo, ya parcialmente puestas en práctica, sería el conjunto de iniciativas que surgieron en el municipio de Lousã. Esta localidad ha elegido el "turismo accesible" como estrategia de desarrollo de un destino turístico de excelencia para convertirlo en un proyecto más ambicioso?.

El municipio de Lousã, localizado en la región Centro de Portugal, se integra en la red de las Aldeias do Xisto (constituida por cinco pueblos), y es actualmente consi-

9 El proyecto está siendo coordinado por el Dr. Filipe Carvalho, quien gentilmente cedió información sobre el mismo. Ver igualmente http://www.cm-lousa.pt/turismo/turismo_acessivel_esp.htm 
derado como un destino de Turismo de Naturaleza, Rural y Activo. A partir de ahora, pretende ser también reconocido como el primer destino de Turismo Accesible en Portugal, a través del proyecto "Lousã, Destino de Turismo Accesible". Con todo, la preocupación por la accesibilidad y la inclusión social no surgió solamente como estrategia de desarrollo turístico, sino como marca de la sociedad civil de este municipio que, desde hace muchos años, ha desarrollado múltiples iniciativas de orden público y voluntario. Así, por ejemplo, en 2004 se creó en el Ayuntamiento la Provedoria Municipal $^{10}$ de las Personas con Discapacidad de Lousã, mostrando de esta manera la importancia especial que este tema tenía para el municipio. El tema se fue integrando progresivamente en la estrategia general de desarrollo de la localidad, habiendo sido también adoptado por el sector turístico. Fue de esta forma como Lousã destacó en el panorama portugués, al convertirse en sede del I Congreso Nacional de Turismo Accesible en 2007, "consumando una voluntad de asociar a las potencialidades turísticas del consejo un trabajo de fondo en los dominios de la rehabilitación y de la inserción social preconizado a lo largo de los últimos años por los agente sociales de Lousã” (Câmara Municipal da Lousã, 2009).

Teniendo como ambición ser el primer destino de Turismo Accesible en Portugal, el Ayuntamiento de Lousã ha asumido como apuesta estratégica el proyecto "Lousã, Destino de Turismo Accesible", cuyo programa de acciones contempla operaciones de accesibilidad, en la dimensión turística, en varios niveles, a saber:

- En las unidades de alojamiento, mejorando la accesibilidad física a todas las instalaciones comunes, así como al interior de las habitaciones especialmente adaptadas (las iniciativas abarcan desde un hotel de 4 estrellas hasta unidades de turismo en un espacio rural o un albergue juvenil);

- Mejorando la accesibilidad a los establecimientos de restauración y similares;

- Adaptando y hasta introduciendo nuevas iniciativas de animación, como las actividades outdoors organizadas para deficientes motores.

Por otro lado, el municipio fortalece su apuesta a través de iniciativas en todos los dominios de la vida comunitaria, vía acciones en la vertiente social (integrando servicios de seguridad, salud y apoyo humano, dirigido tanto a la población local como a los visitantes), y por su actuación a nivel territorial (vía intervención en los principales polos de atracción turística: accesibilidad de los equipamientos públicos y desarrollo de nuevas respuestas de transporte adaptado).

Se realizaron ya varias iniciativas que ilustran el empeño del municipio por esta causa, entre las que destacamos las siguientes:

- Se creó el sello "Lousã Acessível" (Lousã Accesible), que se concede a establecimientos que se han vuelto accesibles a personas con discapacidad, contando ya actualmente con 108 establecimientos adheridos. Este sello no representa solamente una herramienta de identificación de las ofertas accesibles, sino que puede

10 Especie de gabinete de apoyo, con una misión propia, integrado en la estructura del Ayuntamiento. 
ser igualmente utilizado como instrumento de promoción, señal de inversión seria en una causa destacada, reveladora de una imagen de responsabilidad social.

- Se proyectó el itinerario "Trilho do Espigão Acessível", una ruta a pie accesible para la observación de los venados, basada en las particularidades de la vida salvaje del destino y de la capacidad de hacer visible la accesibilidad por medio de inversiones en infraestructuras, equipamiento y personal cualificado y motivado.

- Se realizó una acción de formación sobre la temática de las "Acessibilidades e Design for all", con apoyo de profesionales especializados en la materia y dirigida a los diversos agentes públicos y privados, efectivos y potenciales inversores en el turismo accesible de Lousã.

- Se programó y realizó la escapada “Lousã acessível", un paquete de fin de semana comercializado por la agencia de viajes especializada Accessible Portugal $^{11}$.

Este proyecto, ambicioso y complejo, contempla una vertiente de asesoría técnica asumida por la Estructura de Misión "Lousã Accesible", responsable de la gestión del proyecto "Lousã, Destino de Turismo Accesible". Para la consecución de los objetivos delineados, la Estructura de Misión se hace acompañar de un conjunto de expertos nacionales e internacionales con competencias en materia de turismo accesible.

Institucionalmente, la iniciativa cuenta con el patrocinio de entidades como el Gabinete de la Secretaría de Estado Adjunta y de la Rehabilitación, del Instituto Nacional para la Rehabilitación del Turismo de Portugal (I.P.). Regionalmente, están asociados organismos como la Dirección Regional de Economía del Centro, la Comisión de Coordinación y Desarrollo Regional del Centro, o la Entidad Regional de Turismo del Centro. A nivel internacional, tiene conexiones con la European Network for Accesible Tourism (ENAT).

Para obtener financiación, el Ayuntamiento de Lousã ha realizado tres solicitudes: al Programa Operacional (P.O.) de Potencial Humano (ya aprobado), que permite la instalación del equipo técnico para la creación de contenidos, el intercambio de experiencias, los estudios de accesibilidad, etc.; al P.O. Temático de Valorización del Territorio, para la intervención a nivel de las infraestructuras; y también al Programa de Iniciativa Comunitaria ITERREG-SUDOE, una solicitud conjunta con Ávila y Palma de Mallorca, liderada por Lousã, para la creación de una red de destinos de turismo accesible a nivel europeo y que busca la intervención pública en espacios urbanos.

Existe ya una asociación entre Lousã y Ávila en materia de turismo accesible, y se verifica, efectivamente, una creciente conciencia sobre la relevancia del turismo accesible en muchos actores del sector, tanto públicos como privados (no sólo visible en las conferencias dedicadas al tema y en los discursos políticos, sino también en otros foros de discusión sobre estrategias de futuro para el turismo en Portugal y España ${ }^{12}$ ).

11 Primera agencia de viajes y empresa de animación turística dirigida a personas con discapacidades en Portugal.

12 Como ejemplo, se apuntan las diferentes reuniones de trabajo realizadas, en 2008, entre agentes del sector turístico de la región Centro de Portugal y de la comunidad autónoma de Castilla - León (de España), en el ámbito del proyecto "MIT - Movilidad, Innovación y Territorio", que se encuadraron en las iniciativas de la 


\section{6.- CONCLUSIONES.}

La responsabilidad social de las empresas puede ser entendida como una nueva cultura empresarial basada en la gestión ética y en la responsabilidad de la empresa ante la sociedad como cauce para mejorar la competitividad y la reputación de las empresas, respondiendo así a las crecientes demandas de los consumidores y de la sociedad en general (Nieto y Fernández, 2004; Ruano y Rojas, 2006).

Las empresas turísticas, que operan en un entorno muy competitivo y en un mercado cada vez más experimentado, sofisticado, exigente y, simultáneamente, más educado y sensible a las causas sociales, deben, en ese contexto, estar muy atentas a la evolución del concepto de calidad esperado por los consumidores, muy asociado a sus cambios de valores (Nicolau, 2008; Krippendorf, 1989). La discusión en torno al "turismo sostenible" (Kastenholz y Paul, 2004; Middleton y Hawkins, 1998; OMT, 2004) responde a una preocupación creciente en relación a los impactos negativos del turismo en las áreas de destino en que ocurre, pero integra igualmente una preocupación por una experiencia digna y enriquecedora del turista, de acuerdo con el concepto del turismo como "derecho para todos", en una perspectiva de "turismo social" (OMT, 2005; Devile, 2007). El turismo, al ser no sólo un fenómeno económico sino también ambiental, humano y social, tiene que acompañar a la evolución de la sociedad en dirección a una mayor integración de todos los dominios y dimensiones del "sistema turístico" (Middleton y Hawkins, 1998; Bramwell y Lane, 1993).

El turismo accesible surge así como modalidad de oferta turística integrada que permite a todos disfrutar de experiencias turísticas sin barreras (Burnett y Baker, 2001; OMT, 2005). Esta modalidad de turismo no sólo permite a las empresas una especialización (y, por tanto, una mejor satisfacción del mercado de personas con discapacidad), sino también les permite mostrar una imagen más humana, conseguir una reputación de empresa turística con responsabilidad social, lo cual puede constituir una importante ventaja competitiva en el mercado general y, sobre todo, en el que se muestra sensible a las causas sociales.

Por otro lado, como la experiencia turística es vivida de un modo muy complejo en territorios y sistemas de servicios y atracciones más amplios (Leiper, 1979; Gnoth, 2003; Ashworth y Voogd, 1994; Kastenholz, 2005), una integración de varias ofertas accesibles en un destino turístico globalmente más accesible sería lo ideal. Así, los propios territorios, los pueblos, los municipios, los parques naturales, se volverían más accesibles a un mayor número de ciudadanos.

Comunidad de Trabajo Castilla - León y región Centro de Portugal, fomentando la valorización de la realidad territorial de las dos regiones a través de iniciativas de colaboración. En estas reuniones la accesibilidad de la oferta turística fue reconocida por muchos de los representantes de diversos subsectores y entidades del turismo como una potencial marca de distinción para ofertas de excelencia. Así, los representantes del alojamiento hotelero, rural y del Turismo de Naturaleza, además del enoturismo, demostraron una sensibilidad hacia este asunto, apuntando también la necesidad de, simultáneamente, garantizar la accesibilidad a nivel de cada localidad y de la oferta turística en su conjunto. Surgieron, así, una serie de propuestas que apuntaban a la introducción de los requisitos del turismo accesible en la oferta turística, como marca de calidad distintiva del destino Centro - Castilla y León (Kastenholz et al, 2009). 
Surge de esta forma una apuesta por el turismo accesible como estrategia de cualificación y diferenciación del destino, como la asumida por el municipio de Lousã; una apuesta que ha dinamizado muchas iniciativas y nuevos caminos de colaboración entre diversos agentes (públicos y privados), abriendo ventanas de oportunidades muy interesantes en un contexto de desarrollo de los territorios que busca no excluir a nadie de su uso y disfrute. Todo el dinamismo creado en el municipio y entre los stakeholders involucrados ha alcanzado proporciones que han traspasado las fronteras de la localidad, considerándose el proyecto como innovador en el ámbito nacional y hasta motivador de colaboraciones internacionales (Kastenholz et al, 2009) por una causa que moviliza a un creciente número de personas. Los efectos positivos son, por eso, múltiples, beneficiando tanto a una población - un segmento del mercado anteriormente menos atendido (Daruwalla y Darcy, 2005) -, como a los múltiples actores del destino turístico que se benefician económicamente de un refuerzo de diferenciación y, consecuentemente, de mayor demanda de su oferta. Por otro lado, el destino presenta una visión distinta de desarrollo, de responsabilidad social, generadora de otros muchos apoyos de la sociedad civil, permitiendo así un "círculo virtuoso" en un contexto social y territorial más amplio.

\section{7.- BIBLIOGRAFÍA.}

ARENAS, Daniel (2006): "Responsabilidad, Estrategia y Grupos de Interés", Harvard Deusto Marketing \& Ventas, No 77 (Nov./ Dic.), pp. 34-39.

ASHWORTH, G.; VOOGDT, H. (1994): "Marketing and Place Promotion”, en Gold y Ward (eds.): Place Promotion - The use of publicity and marketing to sell towns and regions, pp. 39-52. John Wiley \& Sons, Chichester.

BRAMWELL, B.; LANE, B. (1993): "Sustainable Tourism: an evolving global approach", Journal of Sustainable Tourism, Vol. 1 (1), pp 1-12.

BUENO CAMPOS, Eduardo (2001): Curso Básico de Economía de la Empresa. Un enfoque de organización. Editorial Pirámide. Madrid.

BUHALIS, D.; EICHHORN, V.; MICHOPOULOU, E.; MILlER, G. (2005): Accessibility Market and Stakeholder Analysis, OSSATE -One-Stop-Shop for Accessible Tourism in Europe. University of Surrey, United Kingdom.

BURNETT, J. J.; BAKER, H. B. (2001): "Assessing the travel-related behaviors of the mobility-disabled consumer". Journal of Travel Research, 40 , pp. 4-11.

CÂMARA MUNICIPAL DA LOUSÃ (2009): Lousã - Destino de turismo acessible, http://www.cm-lousa.pt/turismo/turismo acessivel esp.htm (consulta: 20-03-2009)

CARROLL, Archie B. (1999): “Corporate Social Responsibility. Evolution of a Definitional Construct”, Business \& Society, Vol. 38, No. 3 (September), pp. 268295.

CNAD (2003): Turismo acessível, Turismo para todos: guia de referência para profissionais de turismo - uma resposta às necessidades especiais dos turistas com deficiencia. Cooperativa Nacional e Apoio a Deficientes, Lisboa. 
COMISIÓN DE LAS COMUNIDADES EUROPEAS (2001): Libro verde: Fomentar un marco europeo para la responsabilidad social de las empresas. Bruselas, 18.7.2001. COM(2001) 366 final.

COMITÉ ECONÓMICO E SOCIAL EUROPEU (2006): Parecer do Comité Económico e Social Europeu sobre o "Turismo Social na Europa», 2006/C 318/12:http://eurlex.europa.eu/LexUriServ/LexUriServ.do?uri=OJ:C:2006:318: 0067:0077:PT:PDF

CÓRDOBA, Alejandro (2006): "Lecciones sobre Responsabilidad Social Corporativa", Marketing \& Ventas, $\mathrm{n}^{\circ} 73$ (marzo/abril, 2006), pp. 76-79.

CRANE, A.; MATTEN, D. (2007): Business Ethics. $2^{\text {nd }}$ edition. Oxford, UK.

D’AMORE, L. (1988): Tourism -A Vital Force for Peace, Tourism Management, 9 (2), pp. 151-154.

DARUWALLA, P.; DARCY, S. (2005): "Personal and Societal Attitudes to Disability". Annals of Tourism Research, 32 (3), pp. 549-570.

De KADT (1979): Tourism - passport to development? Perspectives on the social and cultural effects of tourism in developing countries. Oxford University, New York.

DEVILE, E. (2007): Turismo e Desenvolvimento Sustentável. Vol 2, GEOTA - Grupo de Estudos de Ordenamento do Território e Ambiente, Lisboa.

ENGLISH TOURISM COUNCIL (2000): People with disabilities and holiday taking. English Tourism Council, London.

ESCUDERO, Javier (2006): "Responsable y rentable”, Emprendedores, nº 102 (marzo), pp. 58-64.

EUROPEAN COMISSION (2006): Opinion of the European Economic and Social Committee on Social tourism in Europe (2006/C 318/12), http://eurlex.europa.eu/LexUriServ/LexUriServ.do?uri=OJ:C:2006:318:0067:0077:EN:PD F (consulta: 28-02-2008)

FORÉTICA (2002): Responsabilidad Social Empresarial. Informe Forética 2002. Situación en España. Forética, Madrid. En www.foretica.es

FRIEDMAN, Milton (1970): “The Social Responsibility of Business is to Increase its Profits", The New York Times Magazine, September 13, pp. 33.

FUJIURA, G. L.; RUTKOWSKIKMITTA, V. (2001): "Counting Disability". In ALBRECHT, G. L.; SEELMAN, K. D.; BURY, M.: Handbook of Disability Studies, pp. 69-96. Thousand Oaks: Sage Publications.

GALÁN LADERO, M.; GALERA CASQUET, C.; VALERO AMARO, V.; DÍAZ MÉNDEZ, M. (2006): “Cause-related marketing in Spain. A consumer behavior perspective", 35th EMAC Conference Proceedings. Atenas (Grecia), 23-26 de mayo.

GNOTH, J. (2003): “Customer activated services networks: towards a dynamic model for tourism destinations", 32nd EMAC Conference Proceedings, Glasgow: University of Strathclyde, May. 
HENDERSON, J. (2007): “Corporate Social Responsibility and Tourism: Hotel Companies in Phuket, Thailand, after the Indian Ocean Tsunami", International Journal of Hospitality Management, 26, pp. 228-239.

HIGGINS-DESBIOLLES, Freya (2006): “More than an 'industry': The forgotten power of tourism as a social force", Tourism Management, Volume 27, Issue 6 (December), pp. 1192-1208.

KASTENHOLZ, E. (2004): “«Management of Demand» as a Tool in Sustainable Tourist Destination Development”, Journal of Sustainable Tourism, Vol. 12 (5), pp. 388-408.

KASTENHOLZ, E. (2005): "Marketing al servicio de un desarrollo sostenible del destino", Investigación y Marketing, № 87 (Junio), pp. 32-36.

KASTENHOLZ, E.; CARVALHO, F.; PINHO, C.; AZEVEDO, J. (2009): “O projecto MIT - uma iniciativa de colaboração transfronteiriça com preocupações de acessibilidade", Revista de Turismo e Desenvolvimento, Volume 11.

KASTENHOLZ, E.; PAUL, G.W. (2004): “Destination Marketing: Profit- or Non-profit Marketing?", Actas das III Jornadas Internacionais de Marketing Público e NãoLucrativo, Covilhã, Abril.

KOTLER, Philip; LEE, Nancy (2005): Corporate Social Responsibility. Wiley. USA. KRIPPENDORF, J. (1989): "The new tourist - turning point for leisure and travel", Tourism Management, June, p.131-135.

LAMBIN (2000): Marketing Estratégico. 4a edição, Lisboa: McGrawHill de Portugal.

LEIPER, N. (1979): “The Framework of Tourism - Towards a definition of Tourism, Tourist, and the Tourism Industry", Annals of Tourism Research, Vol.VI, n⿳4, pp. 390-407.

LIZCANO ÁLVAREZ, José Luis (2006): "Buen Gobierno y Responsabilidad Social Corporativa", Partida doble, $\mathrm{n}^{\circ} 182$ (noviembre), pp. 20-35.

McGEHEE, N.G.; SANTOS, C.A. (2005): "Social change, discourse and volunteer tourism", Annals of Tourism Research, Volume 32, Issue 3 (July), pp. 760-779.

McWILLIAMS, Abagail; SIEGEL, Donald (2001): “Corporate Social Responsibility: A Theory of the Firm Perspective", Academy of Management Review, Vol. 26, No. 1, pp. 117-127.

MIDDLETON, V. T. C.; HAWKINS, R. (1998): Sustainable tourism: a marketing perspective, Butterworth-Heinemann, Oxford.

NICOLAU, Juan L. (2008): “Corporate Social Responsibility: Worth-Creating Activities", Annals of Tourism Research, Volume 35, Issue 4 (October), pp. 9901006.

NIETO, M.; FERNÁNDEZ, R. (2004): "Responsabilidad Social Corporativa: la última innovación en management", Universia Business Review, 1 Trimestre, pp. 28-39.

ORGANIZACIÓN MUNDIAL DEL TURISMO - OMT (2004): “Conceptos y Definiciones - Desarrollo Sostenible del Turismo” - Definición conceptual (agosto de 2004), http://www.world-tourism.org/sustainable/esp/concepts.htm 
ORGANIZACIÓN MUNDIAL DEL TURISMO - OMT (2005): “Assembleia Geral da Organização Mundial do Turismo”, Organização Mundial do Turismo, 30 Novembro, Dakar, Senegal.

PORTER, M.E. (1985): Competitive Advantage, The Free Press, New Cork.

PUERTA, Juan Felipe (2006): "El caso Iberdrola: RSE, algo más que una operación de imagen”, Marketing \& Ventas, $\mathrm{n}^{\mathrm{o}} 77$ (Nov. / Dic.), pp. 50-54.

RUANO, Diana; ROJAS, Patricio (2006): "Marcas responsables: ¿oportunidad o necesidad?", Harvard Deusto Marketing \& Ventas, No 77 (Nov. / Dic.), pp. 40-43.

SUTER, Tracy A. (1995): Cause - Related Marketing: Current issues and future research directions. In www.sbaer.uca.edu/Research/1995/SWMA/95sma141.htm

VIÑETS, Natàlia (2006): "Responsabilidad Social: clave de fidelización interna y externa en MRW”, Harvard Deusto Marketing \& Ventas, No 77 (Nov. / Dic.), pp. 44-48.

WARTICK, Steven L.; COCHRAN, Philip L. (1985): “The Evolution of the Corporate Social Performance Model", Academy of Management Review, Vol. 10, No. 4, pp. 758-769.

WORLD HEALTH ORGANIZATION - WHO (2007): Global Age-friendly Cities: A Guide. http://www.who.int/ageing/publications/Global_age_friendly_cities Guide_English.pdf (consulta: 9-12-2008). 